\title{
Functional neurological symptoms
}

\author{
J Stone \\ Consultant Neurologist, Department of Clinical Neurosciences, Western General Hospital, and Honorary Senior Lecturer, University of \\ Edinburgh, Edinburgh, UK
}

\begin{abstract}
Functional neurological symptoms refer to neurological symptoms that are not explained by disease. They may also be called psychogenic, nonorganic, somatoform, dissociative or conversion symptoms. The most common functional neurological symptoms are non-epileptic attacks and functional weakness. These are common in neurology and general medical practice, especially in emergency situations, where they can be mistaken for epilepsy or stroke. Many studies have shown that these symptoms often persist, are associated with distress and disability and, in the right hands, have a low rate of misdiagnosis. Physicians are often uncertain how to approach patients with these problems. Are patients making up the symptoms? How can the diagnosis be made confidently? What is the best way to explain the diagnosis to the patient? Does treatment ever help? This review takes readers through these questions with practical tips for avoiding common pitfalls, both in diagnosis and management. There is no good evidence that these symptoms are any more 'made up' than irritable bowel symptoms or chronic pain. The diagnosis should usually be made by a neurologist on the basis of positive signs of inconsistency such as Hoover's sign or the typical features of a non-epileptic attack. A 'functional' model of the symptoms is useful both in thinking about the problem and when explaining the symptoms to the patient. There are many useful steps in management that do not require a detailed understanding of aetiology in an individual patient.
\end{abstract}

Correspondence to J Stone, Department of Clinical Neurosciences, Western General Hospital, Crewe Rd, Edinburgh EH4 2XU, UK tel. +44 (0) I3I 537 II67 e-mail jon.stone@ed.ac.uk

KEYWORDS Conversion disorder, functional neurological symptoms, malingering, non-epileptic, non-organic, psychogenic

DECLARATION OF INTERESTS No conflict of interests declared.

Around one-third of neurology outpatients have symptoms that neurologists rate as only 'somewhat' or 'not at all' explained by disease. Around $20 \%$ of patients brought into hospital in apparent status epilepticus and about one in seven patients attending a 'first fit' clinic have a diagnosis of dissociative (non-epileptic) attacks. Patients with functional weakness are at least as common as patients with multiple sclerosis and represent the leading misdiagnosis in patients wrongly given thrombolysis for presumed stroke. A recent study of 3,781 new neurology patients in Scotland found that around $5 \%$ had a primary diagnosis of a functional motor or sensory symptom such as non-epileptic attacks, functional weakness or functional movement disorder. ${ }^{1,2}$

\section{DIAGNOSIS}

A diagnosis should not be made based on the history alone. That is usually done more reliably on the basis of the examination or observation of attacks. The diagnosis should never be made just because investigations are normal. Nonetheless, there are common features:

- Multiple symptoms, especially pain and fatigue.

- A history of other functional symptoms or syndromes, including irritable bowel syndrome, chronic fatigue syndrome, menorrhagia (especially early hysterectomy), fibromyalgia or other poorly explained chronic pain syndromes. 'Brittle asthma', 'atypical chest pain', 'unexplained palpitations' are red flags for anxiety and panic.

- Not all patients with functional symptoms are depressed or anxious, although many are. To find out about current mood symptoms, ask patients if the symptoms make them feel down or worried (rather than asking if they are depressed).

- Only around one in five patients with functional neurological symptoms believes that stress is relevant, whereas patients with disease often think it is. ${ }^{3}$

- Patients with non-epileptic attacks commonly have panic symptoms prior to their attacks ${ }^{4}$ (but are usually reluctant to discuss this as they fear being told they are 'crazy'). The same is often true for patients with acute functional weakness.

- Information about the diagnosis of functional movement disorders and visual symptoms is provided elsewhere..$^{2,5,6}$

\section{FUNCTIONAL WEAKNESS}

Patients with functional weakness may have the following positive signs which help identify the weakness as internally inconsistent and therefore not due to a structural disease. Caution should be exercised 


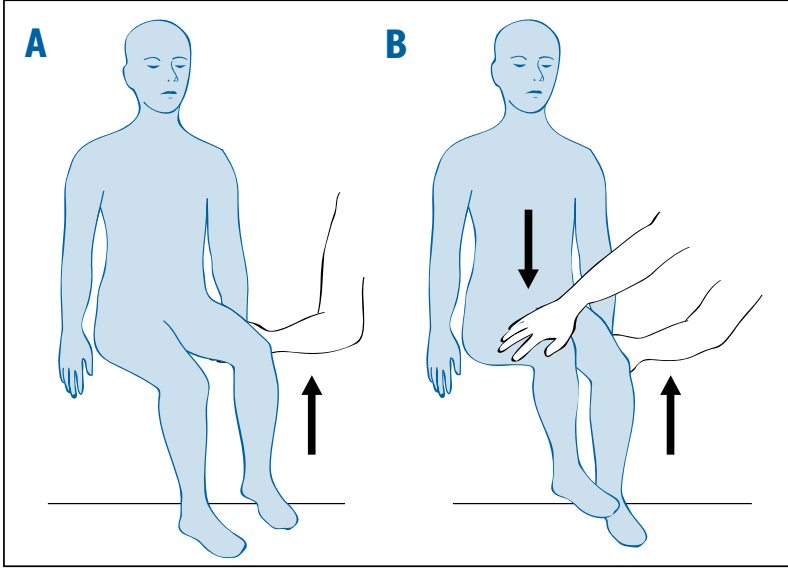

FIGURE I Hoover's sign for functional weakness - a positive physical sign.

A: Weakness of left hip extension.

B: Right hip flexion tested against resistance. Left hip extension becomes strong.

as no physical sign is foolproof, especially in the acute situation:

- Hoover's sign (Figure I). In this test, best carried out with the patient seated, weakness of hip extension returns to normal with contralateral hip flexion against resistance.

- Dragging gait. Patients with acute functional weakness may drag their whole leg behind them with the hip externally or internally rotated (unlike patients with organic hemiparesis who tend to swing or circumduct their leg).

Some common but less reliable indicators are:

- Global pattern of weakness. If someone has a left hemiparesis due to an upper motor neuron lesion then the weakness should be pyramidal in distribution (i.e. extensors weaker than flexors in the arm and flexors weaker than extensors in the leg). Global weakness suggests functional weakness.

- Collapsing weakness. The limb appears to have normal power but collapses at a slight touch (or sometimes even before it has been touched). Beware that pain and misunderstanding can make this sign appear positive when it is not.

- In an acute medicine setting, look out for patients with apparent multiple 'transient ischaemic attacks' over several months, especially if they present with dense weakness and a normal scan. This could be migraine but may be functional.

\section{DISSOCIATIVE (NON-EPILEPTIC) ATTACKS}

Here the diagnosis rests on the nature of the attacks. ${ }^{7,8}$ A wide variety of signs have been recorded to attempt to distinguish non-epileptic attacks from epilepsy (Table I). Ideally, the attack should be captured using video electroencephalography (EEG), but sometimes a recording
TABLE I Selected clinical features that are helpful and unhelpful in distinguishing dissociative (non-epileptic) attacks from epilepsy

\begin{tabular}{|c|c|c|}
\hline & $\begin{array}{l}\text { Dissociative } \\
\text { attacks }\end{array}$ & $\begin{array}{l}\text { Epileptic } \\
\text { seizures }\end{array}$ \\
\hline \multicolumn{3}{|l|}{ Helpful } \\
\hline Duration over 2 minutes & Common & Rare \\
\hline Eyes and mouth closed & Common & Rare \\
\hline Resisting eye opening & Common & Very rare \\
\hline $\begin{array}{l}\text { Visible large bite mark on side } \\
\text { of tongue/cheek/lip }\end{array}$ & Very rare & Occasional \\
\hline Fast respiration during attack & Common & Ceases \\
\hline Grunting/guttural 'ictal cry' sound" & Rare & Common \\
\hline Weeping/upset after a seizure & Occasional & Very rare \\
\hline $\begin{array}{l}\text { Recall for period of } \\
\text { unresponsiveness }\end{array}$ & Common & Very rare \\
\hline Thrashing, violent movements & Common & Rare \\
\hline Post-ictal stertorous breathing & Rare & Common \\
\hline Attacks in medical situations & Common & Rare \\
\hline \multicolumn{3}{|l|}{ Unhelpful } \\
\hline Stereotyped attacks & Common & Common \\
\hline Attack arising from sleep & Occasional & Common \\
\hline Aura & Common & Common \\
\hline Incontinence of urine or faeces & Occasional & Common \\
\hline Injury & Common & Common \\
\hline Report of tongue biting & Common & Common \\
\hline
\end{tabular}

"This is a characteristic fragmented cry caused in a generalised tonic clonic seizure by a tonic diaphragm forcing air against a tonic or clonic glottis.

from a relative's mobile phone is sufficient to be confident of the diagnosis. Patients with non-epileptic attacks commonly end up on anticonvulsants or with pacemakers because no one has considered the diagnosis. In an acute medicine setting physicians should be particularly wary of patients who have seizures arising from general anaesthetic. This is usually a non-epileptic attack. Likewise, always consider the diagnosis for status epilepticus since long-duration attacks unresponsive to (or probably made worse by) diazepam are typical. Studies of the way patients describe their symptoms may also provide useful diagnostic information. For example, patients with dissociative attacks often do not attempt to describe the attack, whereas patients with epilepsy often do. ${ }^{9}$

\section{ARE THEY REALLY ILL?}

Remarkably, the presence or absence of a neurological disease does not seem to make much difference to selfrated measures of disability. In our own study of 3,78I patients, those with symptoms rated as 'not at all explained' by disease were marginally more disabled and significantly more distressed than those whose symptoms were 'completely explained' by disease.' One might 
argue that these kinds of problems are best left alone since they will probably get better anyway. Unfortunately, virtually every follow-up study of functional neurological symptoms shows a persistence of symptoms in the majority of patients in the long term. Rates of medical retirement, consumption of health service resources and use of disability benefits indicate that this is a major public health problem predominantly affecting people of working age.

\section{IS THE DIAGNOSIS WRONG?}

If someone is paralysed and in a wheelchair it is reasonable to wonder how often the diagnosis of functional neurological symptoms is a mistake - was it multiple sclerosis all the time? A systematic review of studies of misdiagnosis found that around $5 \%$ of patients had the wrong diagnosis after an average of five years. ${ }^{10}$ This is the same rate of misdiagnosis as most neurological and psychiatric conditions. However, the diagnosis is not easy and it is usually prudent to ask a neurologist to confirm whether it is correct. Mistakes occur, particularly in the following situations:

- A diagnosis by a non-neurologist.

- Too much emphasis placed on psychiatric history.

- A diagnosis made just because something looks 'weird', without clear evidence of inconsistency on examination.

- The patient has both functional symptoms and an underlying disease.

- The presenting symptom is an abnormality of gait.

- The diagnosis is actually frontal lobe epilepsy.

\section{IS THE PATIENT MAKING UP THE SYMPTOMS?}

Many doctors find themselves asking a question diametrically opposed to their anxiety about 'misdiagnosis': 'How can we be sure that the patient is not making up his or her symptoms?' The simple answer is: 'You can't be sure.' The only definitive ways would be using video surveillance or a confession, which are hardly likely to occur in routine practice. So, instead one has to rely on a number of pieces of circumstantial evidence:

- The similarity of patients' reports of symptoms (around the world);

- The similarity of psychological associations such as adverse childhood experience;

- The persistence of symptoms in follow-up studies;

- The desire for investigations;

- The presence of wear marks on patients' shoes;

- The way in which patients with non-epileptic attacks will often have attacks during an EEG, even when forewarned that if the EEG is normal this means they probably do not have epilepsy.
In my National Health Service (NHS) practice I do rarely meet patients with factitious disorder who are consciously simulating symptoms for medical care. I have also come across patients malingering (for money) in medico-legal practice. In my NHS practice, however, I have stopped worrying about this issue. Even when I see a patient exaggerating I ask myself whether they are doing this to convince me that there is a problem or deceive me about a problem that is not there.

\section{ARE THESE SYMPTOMS REALLY 'PSYCHOGENIC'?}

Most models of why these symptoms happen include biological as well as psychological and interpersonal (social) factors. Adverse life experiences, including childhood adverse experience, personality and emotional disorders can play a role in predisposing someone to symptoms, but not all patients have these factors. Symptoms may be triggered by a panic attack, injury or pain. Often dissociative symptoms, such as depersonalisation (a feeling that your body is not part of you) or derealisation (a feeling of disconnection from the world), may be present. Illness beliefs, especially anxiety about a serious cause or the consequences of recurrent attacks or about being labelled as 'psychosomatic', often stand in the way of an effective understanding of the symptoms. Vicious circles of pain, poor sleep, fatigue and disability can put up further barriers to improvement. Functional imaging studies are helping to understand the neural correlates of these symptoms" and that they have a 'biology' as well as a 'psychology'. Ultimately, there is no 'one size fits all' aetiological theory and every patient has to be assessed individually, but perpetuating factors provide the best target for treatment.

\section{TREATMENT}

Physicians are in a key position to both help and harm patients with functional neurological symptoms. Giving a constructive diagnosis need not be time-consuming and a successful explanation of the diagnosis can in itself be highly therapeutic. On the other hand, a poor explanation often leads to an angered patient who will be harder to help later on. I have outlined an approach elsewhere, ${ }^{12}$ which involves the following steps:

- Explain to the patient what they do have. I personally use the terms 'functional weakness/movement disorder' and 'dissociative attacks'. Use psychogenic if you wish, but be aware that for most patients words such as 'psychosomatic' and 'psychogenic' mean 'making it up'.

- Explain why you are making the diagnosis. I show patients their Hoover's sign or discuss why their attacks can only be dissociative. This gives the diagnosis more authority and logic.

- Explain what they do not have, e.g. multiple sclerosis, epilepsy, and why. 
- Tell patients you believe them: 'I don't think you are making up or imagining these symptoms/going crazy'.

- Emphasise that the condition is common: "I see lots of patients with similar symptoms.'

- Emphasise the potential for reversibility: 'Because the nervous system is not damaged these symptoms have the potential to improve.'

- Metaphors may be useful: 'This is like a software problem with the brain rather than a hardware problem.'

- Introduce the role of psychological factors: 'This problem is not "all in your mind", but the way you think about things can affect it. So let's think about your Hoover's sign. When you were thinking hard about moving your weak leg it did not work, but then when you were distracted by moving your good leg, your weak leg moved normally.'

- Use written information such as that provided at www.neurosymptoms.org (a self-help website by the author) or www.neadtrust.co.uk. I personally always copy my letters to patients as well.

- Do not get drawn into discussions about 'why' the symptoms have happened - at this early stage it is just too difficult to have much idea about why individuals have particular symptoms. This topic can be revisited later, if necessary. The priority is establishing confidence in the diagnosis.

- Involve family and friends - they need to understand what these symptoms are as well.

Explanation can go a very long way in these symptoms. For persistent symptoms physiotherapy, using encouragement, distraction techniques and graded exercise, can be useful. Referral to a liaison psychiatrist may be an important step, but beware of referrals to uninterested psychiatrists who may reply 'no psychiatric disorder'. Cognitive behavioural therapy exploring patterns of thinking and behaviour over a longer period of time can also be helpful: currently, only dissociative attacks have a clear evidence base for this kind of treatment. ${ }^{13}$ Other forms of psychotherapy may also be useful but need to be conducted by someone who is familiar with functional neurological symptoms.

Many patients do not improve despite treatment and it is important to know when to stop trying. In my own practice if I can help at least one in four chronic patients then I regard that as a reasonable 'number needed to treat'. There is little evidence regarding antidepressants in functional neurological symptoms, but they can be used when there are symptoms that are known to respond to these agents such as depression, anxiety, pain or insomnia.

\section{KEY POINTS}

- Functional neurological symptoms are common and include functional weakness and dissociative (nonepileptic) attacks.

- Misdiagnosis, concern about malingering or a perception that symptoms typically resolve spontaneously are all unfounded reasons why doctors ignore these patients.

- Use positive evidence based on the physical examination (e.g. Hoover's sign) or features of the attack to make the diagnosis, but seek a neurological opinion for clarification.

- Explanation should initially focus on clearly naming the diagnosis, explaining why you have made the diagnosis and that you believe the patient. Do not be tempted to explain to a patient why it has happened.

- Not everyone benefits from treatment, but more than enough do to make it worthwhile trying.

\section{REFERENCES}

I Stone J, Carson A, Duncan R et al. Symptoms 'unexplained by organic disease' in II44 new neurology out-patients: how often does the diagnosis change at follow-up? Brain 2009; 132:2878-88. doi:10.1093/brain/awp220

2 Hallett M, Fahn S, Jankovic J et al., editors. Psychogenic movement disorders: neurology and neuropsychiatry. Philadelphia: Lippincott Williams \& Wilkins; 2006.

3 Stone J, Warlow C, Sharpe M. The symptom of functional weakness: a controlled study of 107 patients. Brain 2010 I33:15375I. doi: 10.1093/brain/awq068

4 Goldstein LH, Mellers JD. Ictal symptoms of anxiety, avoidance behaviour, and dissociation in patients with dissociative seizures. J Neurol Neurosurg Psychiatry 2006; 77:616-21. doi:10.1136/ jnnp.2005.066878

5 Chen CS, Lee AW, Karagiannis A et al. Practical clinical approaches to functional visual loss.J Clin Neurosci 2007; I4:I-7. doi:I0.1016/j. jocn.2006.03.002

6 Schrag A, Trimble M, Quinn $N$ et al. The syndrome of fixed dystonia: an evaluation of 103 patients. Brain 2004; 127:2360-72. doi:10.1093/brain/awh262

7 Schachter SC, LaFranceWC, editors. Gates and Rowan's nonepileptic seizures. 3rd ed. Cambridge: Cambridge University Press; 2010.

8 Reuber M. Psychogenic non-epileptic seizures: answers and questions. Epilepsy Behav 2008; 12:622-35.

9 Reuber M, Monzoni C, Sharrack B et al. Using interactional and linguistic analysis to distinguish between epileptic and psychogenic nonepileptic seizures: a prospective, blinded multirater study. Epilepsy Behav 2009; 16:139-44. doi:10.1016/j.yebeh.2009.07.018

10 Stone J, Smyth R, Carson A et al. Systematic review of the misdiagnosis of conversion symptoms and 'hysteria'. BMJ 2005; 331:989-9I. doi:I0.I I36/bmj.38628.466898.55

II Vuilleumier P, Chicherio C, Assal F et al. Functional neuroanatomical correlates of hysterical sensorimotor loss. Brain 2001; 124:107790. doi:10.1093/brain/I24.6.1077

12 Stone J. The bare essentials: functional symptoms in neurology. Pract Neurol 2009; 9: I79-89. doi:I0.I I36/jnnp.2009.I77204

13 Goldstein LH, Chalder T, Chigwedere C et al. Cognitive-behavioral therapy for psychogenic nonepileptic seizures: a pilot RCT. Neurology 2010; 74:1986-94. doi:I0.12I2/WNL.0b0I3e3 I8le39658 


\section{SELF-ASSESSMENT QUESTIONS}

I. Which one of the following is true of the diagnosis of functional neurological symptoms?

A. Psychiatric history is the most important feature.

B. The diagnosis must be made on the basis of positive features of inconsistency on examination or incongruity with disease.

C. Normal investigations mean that the problem is likely to be functional.

D. Attacks arising from generalised anaesthesia are usually epilepsy.

E. Adverse life experiences are a universal finding in these patients.

2. Which one of these clinical features suggests a diagnosis of epilepsy rather than a diagnosis of dissociative (non-epileptic) attacks?

A. Report of tongue biting.

B. Aura.

C. Urinary incontinence.

D. Eyes closed tightly throughout attack.

E. Grunting guttural cry at the onset of the attack.

3. In the diagnosis of functional weakness which one of the following is true?

A. Upper motor neurone weakness is typically global.

B. Functional hemiparetic gait is usually of a 'circumducting' type.

C. Hoover's sign means that weakness of ankle dorsiflexion returns to normal with contralateral ankle plantarflexion against resistance.

D. Collapsing weakness is common but may be caused by pain.

E. Multiple episodes of dense hemiparesis usually suggests cerebrovascular disease.
4. In the treatment of functional neurological symptoms which one of the following is not an early priority in explanation?

A. Give the symptoms a name (i.e. functional weakness, dissociative attacks).

B. Tell patients you believe them.

C. Make links between their symptoms and their known history of childhood abuse.

D. Explain that they have the potential for improvement.

E. Explain on what basis you are making the diagnosis e.g. pattern of weakness, etc.

5. Which one of the following statements about functional neurological symptoms is incorrect?

A. Misdiagnosis rates in published studies are about $5 \%$ at five years.

B. Most follow-up studies of functional neurological symptoms show that the majority of patients spontaneously improve.

C. At least $20 \%$ of patients in apparent status epilepticus are having a dissociative (non-epileptic) attack.

D. Functional weakness is one of the most common diagnoses in patients who are mistakenly given tPA for suspected stroke.

E. Factitious disorder means simulating symptoms for medical care (and not money).

This paper was originally published as part of the Neurology module in the RCPE Online Continuing Medical Education Programme. Online CME, including the anwers to these questions, is available to Fellows and Members at: http://www.rcpe.ac.uk

\section{CONTINUING MEDICAL EDUCATION (CME)}

\section{Online distance learning and self-assessment from the RCPE}

To allow users to update their general medical knowledge, the online CME modules contain a variety of material, including articles, interactive cases and quizzes, webstreamed lectures and self-assessment multiple choice questions (MCQs). Each module also includes material of particular relevance to those undertaking core medical training.
CME modules are eligible for external distancelearning Continuing Professional Development (CPD) credits under the UK Federation of Royal Colleges of Physicians CPD scheme. To claim these credits you must take the MCQ test for the module (only available online). Full instructions are given with each module.
The College's online CME programme is available to Fellows, Collegiate Members, Associates and e-Associates. Log on to the Fellows' and Members' secure area at: http://www.rcpe.ac.uk. If you have forgotten your username or password please contact: webmaster@rcpe.ac.uk

\section{Currently available} for CPD points

- Neurology

- Oncology

\section{Coming soon}

- Rheumatology

- Tropical Medicine

- Respiratory

- Public Health 